\title{
BARRERAS PERSONALES PARA EL APRENDIZAJE EN ESTUDIANTES DE BACHILLERATO
}

\author{
Dora Esperanza Sevilla Santo \\ Mario José Martín Pavón \\ Jorge Alberto Ramírez de Arellano De la Peña \\ Sandra Paola Sunza Chan \\ Universidad Autónoma de Yucatán (México)
}

\begin{abstract}
RESUMEN: El artículo deriva de un proyecto que buscó identificar los factores personales que se constituyen en barreras para el aprendizaje de los estudiantes de bachillerato. Se desarrolló desde el paradigma cuantitativo, con alcance correlacional. La muestra la integraron 2669 alumnos de primer año del subsistema de Escuelas Preparatorias Estatales de Yucatán, México. Para la recolección de información se construyó un instrumento en escala tipo Likert que indagó sobre los factores Autoconcepto, Expectativas, Pensamiento reflexivo y Estrategias de aprendizaje. La identificación de los que se constituyen en barreras para el aprendizaje se realizó correlacionando los indicadores respectivos con el rendimiento académico, a través del coeficiente de Pearson. La importancia de estos sobre el aprendizaje se determinó calculando el modelo de regresión por el método Stepwise; siendo los indicadores de importancia los coeficientes de regresión estandarizados. Para determinar si los factores estudiados tenían un efecto conjunto sobre el aprendizaje se utilizó la técnica de análisis discriminante. Los resultados resaltan la importancia del autoconcepto, las estrategias de aprendizaje y el pensamiento reflexivo para el aprendizaje, estos dos últimos insumos indispensables para cumplir con las demandas de los contenidos curriculares; dejándose entrever la necesidad de realizar una propuesta curricular que contemple su desarrollo.
\end{abstract}

PALABRAS CLAVE: dificultad de aprendizaje, rendimiento académico, educación media superior.

\section{PERSONAL LEARNING BARRIERS IN HIGH SCHOOL STUDENTS}

ABSTRACT: This article stems from a project that sought to identify the personal factors that constitute barriers to learning for high school students. It 
was developed from the quantitative paradigm, with a correlational scope. The sample was made up of 2669 first-year students from the Public High Schools subsystem in Yucatan, Mexico. For the collection of information, a Likert scale instrument was constructed that investigated the factors Self-concept, Expectations, Reflective thinking and Leaning Strategies. The identification of those that constitute barriers to learning was done by correlating the respective indicators with academic performance through Pearson's coefficient. The importance of these on learning was determined by calculating the regression model by the Stepwise method; the importance indicators being the standardized regression coefficients. The discriminant analysis technique was used to determine whether the studied factors had a joint effect on learning. The results highlight the importance of self-concept, learning strategies and reflective thinking for learning, the latter two being indispensable inputs to meet the demands of the curricular contents and thus implying the need to make a curricular proposal that contemplates its development.

KEYWORDS: Learning difficulty, academic performance, upper secondary education.

Recibido: 30/04/2019

Aceptado: 03/03/2020

Correspondencia: Dora Esperanza Sevilla Santo, Facultad de Educación, Universidad Autónoma de Yucatán. Merida - Motul, Gran San Pedro Cholul, Cholul, 97305 Mérida, Yucatán (México). Email: dora.sevilla@correo.uady.mx.

\section{INTRODUCCIÓN}

El aprendizaje es un proceso que implica la adquisición de conocimientos y el desarrollo de habilidades, destrezas y actitudes; es un cambio en la capacidad o disposición humana, relativamente duradero que no puede ser explicado por los procesos de maduración (Fuentes, 2018). Bajo esta perspectiva, existen estudios que han tratado de identificar los factores que inciden en el rendimiento académico de los estudiantes y que se constituyen en barreras para su aprendizaje, algunos de los cuales reportan la importancia de los factores vinculados con el contexto, otros con las instituciones educativas y en mayor medida aquellos que aluden a factores que atañen a la persona que aprende.

En este sentido, es que se habla de dos tipos de trayectoria escolar, la teórica y la real. La primera se refiere al recorrido que debe seguir el estudiante y que está determinada por los tiempos marcados por el sistema educativo, considerando que los sujetos ingresan al sistema a la edad establecida, que transitan por el mismo de forma continua sin repetir o abandonar la escuela y concluyen a la edad prevista para el nivel educativo en cuestión, habiendo adquirido los aprendizajes esperados en el perfil de egreso (Marchesi, Blanco y Hernández, 2015). Sin embargo, según Campos, 
Habiaga, Méndez y Presa (2016) en paralelo a las trayectorias teóricas se tienen las trayectorias reales, entendidas como los itinerarios que realizan los alumnos en su escolaridad y que pueden o no coincidir con la trayectoria teórica, cuya discrepancia se caracteriza por la falta de logro de los aprendizajes y que se refleja en el bajo rendimiento académico.

Al respecto, Becerra (2016) señala que los factores que afectan el aprendizaje pueden ser de dos tipos: los propios de las instituciones educativas y los relacionados con los estudiantes; estos últimos pueden agruparse en académicos, psicosociales, demográficos, cognoscitivos y motivacionales. Sobre el tema, Barrios y Frías (2016) señalan que la autodeterminación, la autoeficacia, la autorregulación, el autoconcepto, la espiritualidad, las habilidades sociales, la identidad positiva y la habilidad cognitiva, son características que favorecen el aprendizaje de quien las posee.

En México, la Secretaría de Educación (2017) identifica en un diagnóstico realizado que los problemas socioeconómicos, así como la falta de orientación vocacional, de interés en los estudios y otras situaciones como la violencia y los embarazos no deseados propician el bajo rendimiento académico de los estudiantes y el abandono escolar.

De manera específica, existe literatura que sustenta que el autoconcepto es uno de los factores que más influye en el aprendizaje (Ibarra y Jacobo, 2016; Chacón et al., 2017), refiriendo que cuando el estudiante tiene un autoconcepto positivo muestra una actitud propositiva frente a las demandas que la escuela le requiere, es participativo en las clases, entabla buenas relaciones con profesores y compañeros y se siente motivado para aprender. También señalan que desde los contextos educativos se puede promover el desarrollo de este factor, para lo cual es necesario brindarles una retroalimentación adecuada de su actuar en las clases y en las tareas y evaluaciones, para que sea capaz de reconocer sus fortalezas.

Otro estudio que analiza factores personales es el realizado por Jaquinet, Rivero y Garnache (2016), quienes comentan que el alumno aprende dependiendo de su motivación, la cual está influenciada por los estados emocionales positivos, la construcción de una autoestima realista (con apoyo del profesorado y los compañeros de clase), las creencias, los intereses, las metas, el conocimiento sobre el programa educativo en el que se encuentra y los hábitos de estudio.

De igual forma, la literatura reporta como un condicionante importante del aprendizaje a la autoestima, al respecto Laguna (2017) identifica que existe una relación significativa entre este factor y el rendimiento académico, siendo que a los alumnos con alta autoestima les gustan los retos que les plantea el proceso de aprendizaje, son más participativos y comprometidos con lo que sucede en las clases.

Algo semejante señalan Barrios y Frías (2016), al afirmar que una barrera para el aprendizaje es el desequilibrio entre el desarrollo físico y emocional de los estudiantes de bachillerato; del mismo modo, Diesktra (2018) menciona la importancia del desarrollo social y emocional desde la infancia, ya que aquellas personas que no aprenden a regular sus emociones tendrán problemas para interactuar con su ambiente afectando entre otras áreas la académica y laboral. También, Escudero 
(2018) destaca la importancia de los factores socioemocionales; enfatizando que la motivación afecta el aprendizaje a la vez que éste incide en los aspectos socioemocionales.

Otro estudio que destaca la influencia de los factores personales es el desarrollado por Sánchez-Sandoval y Verdugo (2016), quienes señalan que la falta de metas académicas (expectativas) y la carencia de estrategias de aprendizaje efectivas, se constituyen en barreras para el aprendizaje, de manera particular durante la adolescencia; siendo que estos factores logran explicar el $30 \%$ de la variabilidad de este constructo, vislumbrándose la importancia de analizar tanto los factores cognitivos como los no cognitivos. De igual forma, la OCDE (2019) resalta que cuando los estudiantes tienen expectativas vinculadas con su aprendizaje, buscan formas más asertivas de hacer frente a los retos que se les presenten, son más persistentes y en general muestran una actitud positiva hacia su proceso formativo; siendo estas metas las que dan sentido a sus aprendizajes, motivándolos a esforzarse por alcanzarlas.

En cuanto a los factores cognitivos y su relación con el aprendizaje, Barrios y Frías (2016) mencionan que la falta de madurez intelectual es un elemento que se constituye en barrera para el aprendizaje; por lo que desde la perspectiva de los autores del presente artículo ésta podría ser una de las causas que propicia que sea en los primeros semestres de bachillerato en donde se presentan mayores porcentajes de reprobación y deserción.

En la misma línea, Acevedo (2016) demostró que las estrategias de aprendizaje y metacognitivas son elementos esenciales para garantizar el éxito académico de los estudiantes a través de la autorregulación y la planificación, ayudándolos a controlar su proceso formativo, evaluándolos para detectar posibles fallos y transfiriendo todo esto a una acción o situación de aprendizaje. Específicamente, en cuanto a las estrategias de aprendizaje, Parlan, Ibnu, Rahayu y Suharti (2018) señalan que la falta de éstas propicia que el estudiante no logre regular su proceso educativo; al no identificar lo que tienen que hacer para aprender y las exigencias que tienen las tareas que se les encargan.

Bajo esta perspectiva, Pineda y Cerrón (2015) señalan que el pensamiento crítico es el proceso cognitivo que usa el conocimiento y la inteligencia para analizar un tema, que resulta imperativo para el cumplimiento y desarrollo de las actividades académicas. Sin embargo, estos autores advierten que son pocos los alumnos que desarrollan éste, lo que se evidencia con la poca participación en clase, la falta de argumentos en sus aportaciones y la recepción pasiva de la información.

Como puede verse, la preocupación por identificar las barreras que limitan el aprendizaje de los estudiantes no es un tema nuevo; sin embargo, éste permanece vigente dado los elevados índices de deserción y rezago que se presentan; además de que dichas barreras cambian dependiendo del contexto de las instituciones educativas. De aquí que éstas deban de estar preparadas para atender las especificidades de los estudiantes, a fin de que estos logren mejorar su desempeño en la escuela. 


\section{Planteamiento del problema}

Entre los problemas que más afectan los sistemas educativos se encuentran el de la reprobación y el de la deserción escolar; fenómenos que se agudizan en el nivel medio superior, siendo compleja su atención dadas sus múltiples causas. Al respecto, la Comisión Europea (2015) y la Oficina Europea de Estadística (Eurostat, 2015, 2016) consideran que de no atenderse esta problemática, se tendrán repercusiones en la incorporación al campo laboral y a la educación superior.

De acuerdo con datos de la OCDE (2017), en promedio solo el 12\% de los jóvenes de los países que integran este organismo concluyen la educación media superior. De manera específica, en México el Reporte de la Encuesta Nacional de Deserción en la Educación Media Superior (SEP, 2012) señala que la deserción escolar en este nivel pasó de 19.3\% en el ciclo escolar 1994-1995 a 14.4\% en el 2011-2012, lo que representa una disminución en casi una década de tan solo el $4.9 \%$.

Asimismo, según el Instituto Nacional para la Evaluación de la Educación (INEE, 2015), el 35\% de los jóvenes entre 15 y 17 años no se matricularon en el nivel medio superior, siendo éste el nivel educativo obligatorio con el menor número de matrícula. Además, es también en este nivel donde se presenta el mayor rezago educativo (7.3\%); así como el porcentaje más alto de alumnos con extraedad grave (14.9\%). Del mismo modo, al 2014, la eficiencia terminal fue de 63\%, en comparación con $96 \%$ y $88 \%$ de primaria y secundaria respectivamente. También el INEE (2018; citado por Juárez, 2018) señala que aproximadamente el $30 \%$ de los adolescentes entre 15 y 17 años se encuentra fuera de la escuela y de cada diez que ingresan, solo siete terminan en tiempo el bachillerato.

En esta misma línea, un estudio realizado por UNICEF (2018), señala que en México de los dos millones de jóvenes en edad de cursar el bachillerato que se encuentran fuera de la escuela el $1.19 \%$ nunca han estado en este nivel educativo, siendo que cerca del $50 \%$ de estos concluyó la secundaria en tiempo y forma; aludiendo que las causas del abandono y rezago escolar son consecuencia de la interacción entre las condiciones complejas de vida que tienen los adolescentes y la oferta educativa que no considera los aspectos contextuales en los que estos alumnos se encuentran.

Para el caso concreto de Yucatán, el porcentaje de deserción es superior a la media nacional al ubicarse en $17.8 \%$ (en el ciclo escolar 2013-2014); en cuanto al porcentaje de matriculación, en el 2015, fue del $65.9 \%$; siendo que solo el $55.7 \%$ lo realizó de forma oportuna (de acuerdo a la edad). En este mismo análisis, el INEE (2015) muestra que "Yucatán, Veracruz, Oaxaca, Chiapas y Guerrero, en todos los grupos de edad, registraron los mayores porcentajes de rezago grave" (p. 336). Finalmente, Yucatán también se sitúa entre los estados con el porcentaje de eficiencia terminal más bajo del país al ubicarse en $57.5 \%$.

Esta situación ha hecho que las autoridades educativas, realicen acciones concretas por atender estas problemáticas implementando estrategias como el otorgamiento de becas y equipos de cómputo, así como de programas de apoyo como el Programa Construye T (SEP, 2019), entre otras acciones; sin embargo, estos índices siguen siendo altos, lo que los ha llevado a cuestionarse sobre qué es lo que ocasiona que los estudiantes no aprendan. 
Es así que el presente estudio parte de la premisa de que es prioritario analizar las características con las cuales Ilegan los estudiantes al bachillerato, para que atendiéndolas se disminuyan sus efectos en el proceso formativo; siendo en este marco que se buscó dar respuesta al siguiente cuestionamiento:

¿Cuáles son los factores personales que se constituyen en barreras para el aprendizaje en los estudiantes de bachillerato? Estableciéndose para ello como objetivo "Identificar los factores personales que se constituyen en barreras para el aprendizaje en los estudiantes de bachillerato".

\section{Método}

El estudio del cual deriva el presente artículo se desarrolló bajo el paradigma cuantitativo. Con relación a su alcance, éste corresponde al de un estudio correlacional, ya que se buscó establecer la influencia de los factores personales en el aprendizaje de los estudiantes, caracterizado por su rendimiento académico. De acuerdo a la temporalidad de la recolección y análisis de la información, éste clasifica como un estudio transeccional ya que dichas actividades se realizaron en un solo momento (Cohen, Manion y Morrison, 2011).

\section{Participantes}

La población en estudio estuvo conformada por todos los estudiantes de primer año del Subsistemas de Escuelas Preparatorias Estatales de Yucatán inscritos al ciclo escolar 2017-2018 (N=3032). La muestra se determinó a través de un muestreo estratificado, teniendo como marcos de muestreo la relación de las 12 escuelas pertenecientes al subsistema en estudio y la relación de grupos de primer año en cada una de ellas, quedando constituida por 2669 alumnos que se distribuyeron como se presenta en la Tabla 1.

Tabla 1. Distribución de participantes por escuela

\begin{tabular}{|c|c|c|}
\hline Escuela & Frecuencia & Porcentaje \\
\hline 1 & 195 & 7.3 \\
\hline 2 & 217 & 8.1 \\
\hline 3 & 501 & 18.8 \\
\hline 4 & 180 & 6.7 \\
\hline 5 & 115 & 4.3 \\
\hline 6 & 211 & 7.9 \\
\hline 7 & 199 & 7.5 \\
\hline 8 & 541 & 20.3 \\
\hline 9 & 142 & 5.3 \\
\hline 10 & 230 & 8.6 \\
\hline 11 & 79 & 3.0 \\
\hline 12 & 59 & 2.2 \\
\hline Total & $\mathbf{2 6 6 9}$ & $\mathbf{1 0 0}$ \\
\hline
\end{tabular}


La edad promedio de los participantes fue de 15 años cuatro meses, con una variación promedio inferior a un año, por lo que corresponde a la edad normativa para el nivel educativo en estudio. Con relación a su nivel socioeconómico, este se ubica entre el nivel medio y medio bajo, lo cual es consistente al tipo de escuela secundaria de procedencia, dado que 2506 (92.3\%) cursaron este nivel educativo en una escuela pública.

\section{INSTRUMENTOS}

Para la recolección de la información se construyó un cuestionario dirigido a los estudiantes a partir de la revisión de la literatura, encontrándose consistentemente factores personales como: manejo de la TICS, comprensión lectora, razonamiento matemático, pensamiento crítico, habilidad para trabajar en equipo, expectativas hacia la institución, proyecto de vida, autoestima y motivación.

No obstante, considerando el contexto del subsistema se incluyó en el estudio: a) el pensamiento reflexivo; dado que la UNESCO (2015) lo coloca entre las siete competencias que los estudiantes del siglo XXI deben de poseer; además de que es una característica establecida en el perfil de ingreso al bachillerato. b) Las expectativas, ya que de acuerdo con Maza (2018), éstas fungen como elemento de decisión en la elaboración de los planes de acción de los estudiantes, por lo que resultan importantes en la determinación del aprendizaje. c) Las estrategias de aprendizaje, cuya inclusión se debe a lo mencionado por García-Valcárcel y Tejedor (2018) quienes resaltan no solo su importancia, sino también su carácter modificable; es decir, la oportunidad de intervención en ellas para que incidan en el aprendizaje. d) El autoconcepto, el cual diferentes autores lo reportan como importante en el proceso de adaptación escolar, logro académico e implicación del alumnado en el proceso de aprendizaje (Inglés et al., 2015).

Una vez definidos los factores a estudiar, se revisaron los instrumentos de Schmidt, Messoulam y Molina (2008) y el AF-5 de García y Musitu (2009) para el caso del autoconcepto; la escala de estrategias de aprendizaje de Román y Gallego (2008) y la escala de Expectativas de Futuro en la adolescencia de Sánchez-Sandoval y Verdugo (2016), así como el trabajo de Calle (2013) sobre pensamiento reflexivo; entre los más importantes.

Integrada la versión preliminar, se procedió a realizar un juicio de expertos contándose con la participación de cinco jueces con experiencia en investigación educativa. La información proporcionada por estos se analizó con base en los criterios establecidos por Barbero, Vila y Suárez (2003). Posteriormente se realizó un estudio piloto, aplicando el cuestionario a un total de 449 alumnos de primer año de una escuela preparatoria con las mismas características que las pertenecientes al Subsistema de Escuelas Preparatorias Estatales. Con los datos recabados se realizó una prueba de discriminación de los reactivos, comparando los puntajes de los estudiantes que obtuvieron una puntuación superior al cuartil 75, con la de los estudiantes que fue inferior al cuartil 25; esta comparación se realizó a través de la prueba t para muestras independientes y el resultado arrojó que los 58 reactivos del instrumento discriminaron. 
Adicionalmente, con la intención de revelar las dimensiones verdaderas en las que se agrupaban los reactivos, se realizó un análisis de factores por el método de componentes principales y la rotación Varimax, acordando considerar como factores aquellos que agruparan al menos tres ítems y que estos tuvieran cargas factoriales mayores o iguales a 0.40. El resultado de este análisis indicó la conformación de cinco factores; siendo el valor del coeficiente de Kaiser Meyer Olkin de 0.868, lo que asegura que el proceso es confiable (ver Tabla 2).

Tabla 2. Análisis de factores

\begin{tabular}{|c|c|c|c|}
\hline Factor & Definición & Ítems & $\begin{array}{c}\text { Coeficiente de } \\
\text { consistencia alfa } \\
\text { de Cronbach } \\
\end{array}$ \\
\hline $\begin{array}{l}\text { Autoconcepto } \\
\text { negativo }\end{array}$ & $\begin{array}{l}\text { En esta dimensión se consideran aquellos as- } \\
\text { pectos que denotan una valoración negativa } \\
\text { del estudiante respecto a su capacidad para } \\
\text { realizar las actividades académicas que se les } \\
\text { encarga y la falta de atributos que contribuyen } \\
\text { a su buen desempeño escolar }\end{array}$ & $1-6$ & 0.779 \\
\hline $\begin{array}{l}\text { Autoconcepto } \\
\text { positivo }\end{array}$ & $\begin{array}{l}\text { En esta dimensión se consideran aquellos as- } \\
\text { pectos que denotan una valoración positiva } \\
\text { del estudiante respecto a su capacidad para } \\
\text { realizar las actividades académicas que se les } \\
\text { encarga y la posesión de atributos que contri- } \\
\text { buyen a su buen desempeño escolar }\end{array}$ & $7-14$ & 0.820 \\
\hline $\begin{array}{l}\text { Estrategias de } \\
\text { aprendizaje }\end{array}$ & $\begin{array}{l}\text { En esta dimensión se hace referencia a las ac- } \\
\text { tividades o herramientas que el estudiante uti- } \\
\text { liza de manera recurrente y que producen un } \\
\text { buen desempeño académico }\end{array}$ & $15-24$ & 0.874 \\
\hline $\begin{array}{l}\text { Pensamiento } \\
\text { reflexivo }\end{array}$ & $\begin{array}{l}\text { Esta dimensión se refiere a las estrategias cog- } \\
\text { nitivas utilizadas por el estudiante que promue- } \\
\text { ven una actitud crítica }\end{array}$ & $25-29$ & 0.805 \\
\hline Expectativas & $\begin{array}{l}\text { Esta dimensión hace referencia a lo que el es- } \\
\text { tudiante espera desarrollar como consecuencia } \\
\text { de la conclusión de sus estudios, tanto en el } \\
\text { ámbito académico como personal }\end{array}$ & $30-33$ & 0.698 \\
\hline
\end{tabular}

Con relación a la confiabilidad del instrumento ésta se calculó a través del alfa de Cronbach, siendo su valor de 0.955 , lo que lo hace un instrumento confiable.

\section{ANÁLISIS DE LA INFORMACIÓN}

En un primer momento, se calcularon indicadores para cada uno de los factores considerados en el instrumento, los cuales se concibieron como el promedio de la suma de las puntuaciones asignadas a los ítems pertenecientes a cada factor. 
Para el caso del rendimiento académico el indicador se calculó a partir de la siguiente fórmula:

$$
I R=\frac{P B+100 * P S}{2} \operatorname{Rep} S+\operatorname{Rep} B-\text { Mrep }
$$

Donde:

$I R=$ Indicador de rendimiento académico

$P B=$ Promedio de las calificaciones de primer semestre de preparatoria

$P S=$ Promedio general de secundaria

$\operatorname{Rep} S=$ Hecho de que el alumno aprobara o no algún grado de secundaria $(1=$ No y $0=\mathrm{Si}$ )

$\operatorname{Rep} B=$ Hecho de que el alumno aprobara o no algún grado de bachillerato (1= No y $0=\mathrm{Si}$ )

Mrep $=$ Número de asignaturas no aprobadas en secundaria

Una vez construidos dichos indicadores, con la intención de determinar los factores personales que influyen en el aprendizaje de los estudiantes (caracterizado por su rendimiento académico), se estudió dicha influencia a través del coeficiente de correlación de Pearson.

Adicionalmente, con la intención de determinar el grado de importancia de cada uno de los factores sobre el aprendizaje, se realizó un análisis de regresión por el método Stepwise; considerándose como variable dependiente al indicador de rendimiento académico y como variables independientes a los indicadores de los factores estudiados.

También, con la intención de determinar si existe un efecto conjunto de los factores estudiados sobre el rendimiento académico de los estudiantes, se aplicó la técnica multivariada de análisis discriminante clasificándose a los estudiantes en dos grupos los de alto rendimiento (aquellos cuya puntuación en el indicador fuera mayor o igual a la media más una desviación estándar) y los de bajo rendimiento (aquellos cuya puntuación en dicho indicador fuera inferior a la media), acordando que el conjunto de factores utilizados para la construcción de la regla discriminante tendría un efecto conjunto si el porcentaje de buena clasificación de dicha regla era de al menos el $80 \%$.

Finalmente, para identificar los aspectos vinculados a los ítems de cada factor que se constituyen en barreras para el aprendizaje de los estudiantes, se calculó la media y la desviación estándar de las puntuaciones asignadas a cada ítem; acordando considerar que aquellos en los que el valor de la media fuera menor a siete representarían dichas barreras (para el caso de los factores autoconcepto positivo, estrategias de aprendizaje, pensamiento reflexivo y expectativas) y mayor a tres puntos para el caso del autoconcepto negativo. 


\section{Resultados}

Con relación a los datos generales de los participantes, se encontró que la edad promedio de los estudiantes fue de 15 años cinco meses con una variación de 10 meses, encontrándose ésta dentro de la edad normativa esperada para el nivel de bachillerato.

Por otra parte, se pudo determinar que el $92.2 \%$ de los estudiantes realizó sus estudios de secundaria en una escuela pública. De igual forma, al cuestionarlos sobre si reprobaron algún grado de secundaria, 126 (4.8\%) indicó que sí; así mismo, se determinó que el promedio de materias reprobadas en secundaria fue inferior a uno. También se encontró que 173 (6.5\%) está recursando el primer grado de preparatoria.

Con la intención de identificar los factores personales que influyen en el aprendizaje de los estudiantes, se analizó la relación de estos con el rendimiento académico a través del coeficiente de correlación de Pearson. Dicho análisis se presenta en la Tabla 3.

Tabla 3. Relaciones de factores personales con el aprendizaje

\begin{tabular}{|l|c|c|}
\hline \multicolumn{1}{|c|}{ Factores de estudio } & r & P \\
\hline Autoconcepto negativo & -0.259 & 0.001 \\
\hline Autoconcepto positivo & 0.287 & 0.003 \\
\hline Estrategias de aprendizaje & 0.244 & 0.003 \\
\hline Pensamiento reflexivo & 0.243 & 0.002 \\
\hline Expectativas & 0.172 & 0.001 \\
\hline
\end{tabular}

Del análisis de la Tabla 3 se observa que todos los factores considerados en el estudio se relacionan con el aprendizaje de los estudiantes.

Adicionalmente, con la finalidad de determinar el grado de importancia que guarda cada uno de los factores estudiados sobre el aprendizaje, se realizó un análisis de regresión siendo los resultados los que se presentan en la Tabla 4.

Tabla 4. Análisis del nivel de importancia de los factores estudiados sobre el aprendizaje

\begin{tabular}{|l|c|c|}
\hline \multirow{2}{*}{\multicolumn{1}{|c|}{ Factor en estudio }} & \multicolumn{2}{c|}{ Estadísticos } \\
\cline { 2 - 3 } & $\begin{array}{c}\text { Coeficiente } \\
\text { de regresión }\end{array}$ & P \\
\hline Constante & 70.676 & 0.002 \\
\hline Autoconcepto negativo & -0.859 & 0.001 \\
\hline Autoconcepto positivo & 0.810 & 0.001 \\
\hline Estrategias de aprendizaje & 0.371 & 0.014 \\
\hline Pensamiento reflexivo & 0.388 & 0.008 \\
\hline Expectativas & 0.302 & 0.026 \\
\hline
\end{tabular}


Como puede apreciarse en la Tabla 4, los factores que resultan más importantes son el autoconcepto negativo, seguido del autoconcepto positivo y el pensamiento reflexivo, siendo que dicho modelo logra explicar el $11.7 \%$ de la variabilidad de las relaciones.

Para complementar el estudio de las relaciones, se buscó determinar si existe un efecto conjunto de los factores estudiados sobre el aprendizaje, utilizándose la técnica multivariada de análisis discriminante clasificando a los estudiantes en uno de dos grupos: estudiantes de alto rendimiento y estudiantes de bajo rendimiento. Los resultados de este análisis se presentan en la Tabla 5.

Tabla 5. Análisis del efecto conjunto de los factores estudiados sobre el aprendizaje

\begin{tabular}{|l|c|}
\hline \multicolumn{1}{|c|}{ Factor en estudio } & $\begin{array}{c}\text { Coeficiente de la fun- } \\
\text { ción discriminante }\end{array}$ \\
\hline Autoconcepto negativo & -0.353 \\
\hline Autoconcepto positivo & 0.506 \\
\hline Estrategias de aprendizaje & 0.318 \\
\hline Pensamiento reflexivo & 0.206 \\
\hline Expectativas & -0.129 \\
\hline Porcentaje de buena clasificación & 75.9 \\
\hline
\end{tabular}

Como puede apreciarse en la Tabla 5, de acuerdo a lo establecido en la metodología, no existe un efecto conjunto de los factores estudiados sobre el aprendizaje.

Por otra parte, con el propósito de identificar los aspectos específicos de cada factor que se constituyen en barreras para el aprendizaje, se realizó un análisis calculando las medias y las desviaciones estándar de las puntuaciones asignadas por los estudiantes a cada ítem. El estudio de dichos aspectos para el factor autoconcepto negativo se presenta en la Tabla 6.

Tabla 6. Aspectos del autoconcepto negativo que se constituyen en barreras para el aprendizaje

\begin{tabular}{|l|c|c|}
\hline \multicolumn{1}{|c|}{ Aspecto evaluado } & $\overline{\mathbf{x}}$ & $\mathbf{S}$ \\
\hline 1. Aunque me esfuerce siempre me va mal en la escuela & 3.1 & 2.8 \\
\hline 2. Cometo muchos errores cuando hago mis tareas & 3.7 & 2.7 \\
\hline $\begin{array}{l}\text { 3. La mayoría de las actividades que proponen los profe- } \\
\text { sores me estresan }\end{array}$ & 4.2 & 3.1 \\
\hline $\begin{array}{l}\text { 4. No puedo hacer bien mis tareas, aunque preste aten- } \\
\text { ción a las explicaciones de mis profesores }\end{array}$ & 2.6 & 3.0 \\
\hline $\begin{array}{l}\text { 5. Participo poco en clase porque no tengo nada impor- } \\
\text { tante que aportar }\end{array}$ & 3.4 & 3.2 \\
\hline 6. Se me olvida fácilmente lo que aprendo & 3.7 & 3.2 \\
\hline
\end{tabular}


Del análisis de la Tabla 6, se observa que de acuerdo a lo establecido en la metodología, el único aspecto del autoconcepto negativo que no se constituye en barrera para el aprendizaje de los estudiantes es el relacionado con el sentimiento de no poder hacer bien las tareas, aunque se preste atención a las explicaciones de los profesores.

De igual forma, en la Tabla 7 se presenta el análisis realizado a los aspectos vinculados al autoconcepto positivo.

Tabla 7. Análisis de los aspectos del autoconcepto positivo que influyen en el aprendizaje

\begin{tabular}{|l|c|c|}
\hline \multicolumn{1}{|c|}{ Aspectos evaluados } & $\overline{\mathbf{x}}$ & $\mathbf{S}$ \\
\hline $\begin{array}{l}\text { 7. Cuando me concentro puedo estudiar cualquier } \\
\text { cosa }\end{array}$ & 7.9 & 2.6 \\
\hline $\begin{array}{l}\text { 8. Soy capaz de participar aportando comentarios o } \\
\text { respondiendo las preguntas de los profesores }\end{array}$ & 6.5 & 2.8 \\
\hline $\begin{array}{l}\text { 9. Soy capaz de entender los diferentes temas que } \\
\text { abordan los profesores durante las clases }\end{array}$ & 6.9 & 2.4 \\
\hline $\begin{array}{l}\text { 10. Me considero capaz de competir académicamen- } \\
\text { te con cualquiera de mis compañeros }\end{array}$ & 6.1 & 3.1 \\
\hline $\begin{array}{l}\text { 11. Soy capaz de relacionar mis ideas con conoci- } \\
\text { mientos adquiridos anteriormente }\end{array}$ & 6.8 & 2.5 \\
\hline $\begin{array}{l}\text { 12. Soy capaz de realizar cualquier trabajo académi- } \\
\text { co que encarguen los profesores }\end{array}$ & 7.4 & 2.4 \\
\hline $\begin{array}{l}\text { 13. Soy un buen alumno, considerando mis cualidades } \\
\text { y conducta en la escuela }\end{array}$ & 7.6 & 2.9 \\
\hline $\begin{array}{l}\text { 14. Soy capaz de mantener un buen rendimiento a lo } \\
\text { largo de todo el semestre }\end{array}$ & 7.0 & 2.5 \\
\hline
\end{tabular}

De la Tabla 7 se observa que los aspectos relacionados con el autoconcepto positivo que se constituyen en barreras para el aprendizaje de los estudiantes, se relacionan con el no sentirse capaces de: participar en la clase respondiendo las preguntas que hacen los profesores, entender los temas o relacionarlos con otros vistos previamente, así como competir académicamente con sus compañeros.

De manera análoga, en la Tabla 8 se presenta el estudio de los aspectos relacionados con el factor estrategias de aprendizaje.

Del análisis de la Tabla 8 se observa que la mayoría de los aspectos vinculados con las estrategias de aprendizaje influyen negativamente en éste, salvo el hecho de buscar aprender cosas nuevas para profundizar después en ellas. 
Tabla 8. Análisis de los aspectos de las estrategias de aprendizaje que influyen en el aprendizaje

\begin{tabular}{|l|c|c|}
\hline \multicolumn{1}{|c|}{ Aspectos evaluados } & $\overline{\mathbf{x}}$ & $\mathbf{S}$ \\
\hline $\begin{array}{l}\text { 15. Busco aprender cosas nuevas para profundizar después } \\
\text { en ellas }\end{array}$ & 7.0 & 2.6 \\
\hline $\begin{array}{l}\text { 16. Acostumbro buscar información a favor y en contra de } \\
\text { mis argumentaciones }\end{array}$ & 5.7 & 2.9 \\
\hline $\begin{array}{l}\text { 17. Uso cuadros sinópticos, mapas mentales, dibujos, etc., } \\
\text { para organizar mis ideas }\end{array}$ & 5.1 & 3.3 \\
\hline $\begin{array}{l}\text { 18. Busco información necesaria para comprender un tema } \\
\text { 19. Antes de entregar mis tareas, elaboro varias versiones } \\
\text { hasta llegar a la que considero que cumple con lo solicitado }\end{array}$ & 6.6 & 2.8 \\
\hline 20. Subrayo las ideas principales de los textos que leo & 6.1 & 3.1 \\
\hline $\begin{array}{l}\text { 21. Busco retroalimentar mis ideas con las opiniones de } \\
\text { otros }\end{array}$ & 5.9 & 2.9 \\
\hline $\begin{array}{l}\text { 22. Reviso los argumentos de mis trabajos antes de redactar } \\
\text { las conclusiones }\end{array}$ & 6.5 & 2.8 \\
\hline $\begin{array}{l}\text { 23. Analizo continuamente mi proceso de aprendizaje para } \\
\text { identificar lo que debo mejorar y por qué }\end{array}$ & 6.4 & 2.8 \\
\hline $\begin{array}{l}\text { 24. Retomo las ideas de los compañeros para explicar lo } \\
\text { visto en la clase }\end{array}$ & 5.9 & 2.8 \\
\hline
\end{tabular}

De igual forma, en la Tabla 9 se presenta el análisis de los aspectos relacionados con el factor pensamiento reflexivo.

Tabla 9. Análisis de los aspectos del pensamiento reflexivo que influyen en el aprendizaje

\begin{tabular}{|l|c|c|}
\hline \multicolumn{1}{|c|}{ Aspectos evaluados } & $\overline{\mathbf{x}}$ & $\mathbf{S}$ \\
\hline $\begin{array}{l}\text { 25. Antes de actuar reflexiono sobre las ventajas y des- } \\
\text { ventajas de lo que voy a hacer }\end{array}$ & 6.5 & 3.0 \\
\hline $\begin{array}{l}\text { 26. En mis escritos corrijo los errores que pueden afec- } \\
\text { tar la claridad }\end{array}$ & 6.9 & 2.8 \\
\hline $\begin{array}{l}\text { 27. Busco respuestas alternativas a los problemas que } \\
\text { se me plantean }\end{array}$ & 6.8 & 2.6 \\
\hline $\begin{array}{l}\text { 28. Me comunico de manera eficaz, reflexionando an- } \\
\text { tes de hablar }\end{array}$ & 6.5 & 2.7 \\
\hline $\begin{array}{l}\text { 29. Mis decisiones las tomo pensando en la manera en } \\
\text { que éstas influirán en mi futuro }\end{array}$ & 7.5 & 2.6 \\
\hline
\end{tabular}

En la Tabla 9 se observa que la mayoría de los aspectos vinculados con el pensamiento reflexivo influyen negativamente en el aprendizaje de los estudiantes, excepto el hecho de que los estudiantes toman sus decisiones pensando en la manera en que éstas influirán en su futuro. 
En cuanto al factor expectativas, en la Tabla 10 se presenta el análisis de los aspectos vinculados con éste.

Tabla 10. Análisis de los aspectos de las expectativas que influyen en el aprendizaje

\begin{tabular}{|l|c|c|}
\hline \multicolumn{1}{|c|}{ Aspectos evaluados } & $\overline{\mathbf{x}}$ & S \\
\hline $\begin{array}{l}\text { 30. Como resultado de mis estudios profesionales me } \\
\text { compraré un carro }\end{array}$ & 7.0 & 3.2 \\
\hline 31. Considero que terminaré mis estudios de bachillerato & 9.0 & 2.1 \\
\hline $\begin{array}{l}\text { 32. Frecuentemente pienso en las cosas que haré en el } \\
\text { futuro }\end{array}$ & 8.8 & 2.1 \\
\hline $\begin{array}{l}\text { 33. Como resultado de mis estudios lograré tener una } \\
\text { casa propia }\end{array}$ & 9.0 & 2.0 \\
\hline
\end{tabular}

Del análisis de la Tabla 10, se observa que ninguno de los aspectos vinculados con las expectativas se constituye en una barrera para el aprendizaje de los estudiantes.

\section{Discusión}

Uno de los primeros hallazgos del presente estudio, se relaciona con la importancia que reviste el autoconcepto como el factor que más influye en el aprendizaje de los estudiantes; lo cual coincide con lo señalado por Barrios y Frías (2016) quienes identifican que valorar de manera positiva la capacidad para realizar las actividades académicas favorece el aprendizaje.

Llama la atención que desde el análisis factorial realizado, con la versión preliminar del instrumento, los ítems correspondientes a dicho factor hayan sido separados en autoconcepto positivo y negativo, siendo este último el que resulta más influyente. Hecho que deja entrever que los estudiantes tienden a reconocer con mayor facilidad sus limitaciones que sus fortalezas, lo que pudiera ser promovido desde las instituciones educativas y el hogar, donde existe una cultura en la que se resaltan más las debilidades que las habilidades que los alumnos poseen. En otras palabras, no se considera lo mencionado por Ibarra y Jacobo (2016) y por Chacón et al. (2017), quienes enfatizan la necesidad de promover el desarrollo de un autoconcepto positivo en los estudiantes, dado que éste los torna más motivados y participativos en las clases al sentirse seguros de sus capacidades.

Para hacer realidad lo anterior, es necesario que el profesor sea capacitado en la detección de aquellos aspectos que influyen tanto positiva como negativamente en el aprendizaje, para que sean tomados en cuenta en la planeación y desarrollo de sus clases, buscando hacer realidad un proceso de enseñanza centrado en el alumno.

Por otra parte, un resultado que debe de alentar el trabajo de las instituciones educativas en relación con la puesta en marcha de estrategias que ayuden a eliminar los obstáculos presentes en el proceso formativo de los estudiantes, es el hecho de que aunque el factor expectativas mostró relación con el aprendizaje de los estudiantes, ninguno de los aspectos vinculados a él se constituye en una barrera para éste, lo 
que permite afirmar que los estudiantes visualizan el cursar el bachillerato como un elemento para tener un mejor nivel de vida.

Al respecto, Sánchez-Sandoval y Verdugo (2016) resaltan la importancia de las expectativas académicas para que los estudiantes le encuentren sentido a sus aprendizajes, bien sea que continúen con sus estudios o se incorporen al mercado laboral. Lo anterior coincide con la visión de organismos internacionales como OCDE (2019) que afirman que es a través de la educación como se pueden alcanzar mejores condiciones de vida. Es decir, desde los contextos educativos debe fomentarse que los estudiantes establezcan metas y expectativas realistas, de tal forma que vean en éstas la motivación para continuar y concluir sus estudios.

Con relación a los factores personales de carácter cognitivo, particularmente en lo referente al factor pensamiento reflexivo, los resultados coinciden con lo que reportan Pineda y Cerrón (2015), así como organismos como UNESCO (2015), quienes resaltan su importancia para afrontar las exigencias de los planes de estudio y como una de las competencias básicas que deben desarrollarse en el bachillerato.

De manera específica, el que la falta de reflexión antes de hablar y actuar, la falta de costumbre de revisar y corregir los errores en las tareas y el no buscar soluciones alternas a los problemas que se les plantean, resulten ser barreras para el aprendizaje de los estudiantes, deja ver la cultura de la inmediatez que envuelve a los estudiantes de estas nuevas generaciones, la cual es propiciada en buena medida por los recursos tecnológicos con los que cuentan, mismos que ponen a su disposición una enorme cantidad de información que utilizan como insumo para el desarrollo de sus actividades académicas; pero que muchas veces no analizan, debido en parte a la falta de un diseño apropiado de las tareas por parte de los docentes, las cuales les permiten cumplir con ésta sin necesidad de apropiarse de los contenidos y de reflexionar sobre la temática estudiada, convirtiéndolos en receptores pasivos de la información (Pineda y Cerrón, 2015). Esta situación podrá llevar a los futuros trabajadores y profesionistas a no contar con los elementos fundamentales para la toma de decisiones que sus puestos laborales les impongan.

En lo que respecta a las estrategias de aprendizaje, el hecho de que casi la totalidad de los aspectos considerados en este factor se constituya en barreras para el aprendizaje, deja ver la clara ausencia de éstas en los estudiantes, algunas de las cuales resultan básicas incluso para niveles educativos previos, lo que habla de la necesidad de desarrollar propuestas curriculares que contemplen su formación y puesta en práctica desde el inicio de la trayectoria escolar. Tal como señalan Acevedo (2016) y Parlan et al. (2018), quienes refieren que cuando los alumnos no tienen estrategias para aprender no sabrán qué, ni cómo hacer lo solicitado en sus clases.

Finalmente, los resultados del estudio, apuntan a la necesidad de realizar investigaciones que analicen la forma en que se implementan los programas de educación obligatoria en México, ya que aunque en todos ellos se establece el desarrollo de un proceso de formación integral centrado en el estudiante; el hecho de que el autoconcepto resultará una de las barreras más importantes para el aprendizaje de los estudiantes, indica que se sigue infravalorando la formación para la vida en favor de la adqui- 
sición de conocimientos, lo que propicia que los estudiantes no le encuentren sentido a sus aprendizajes. De aquí, la necesidad de buscar influir sobre este factor tratando de desarrollar en los alumnos un autoconcepto positivo, a través de la capacitación de los profesores en estrategias de retroalimentación positiva, de modo que esta práctica se centre en identificar las áreas de oportunidad de los trabajos y actividades que los estudiantes realizan en vez de centrarse en criticar a quienes las desarrollan.

De igual forma, se recomienda realizar investigaciones de forma interdisciplinaria (psicólogos y desarrolladores curriculares), a fin de lograr una mejor medición de los factores no cognitivos y el establecimiento de estrategias de intervención que aminoren los efectos nocivos de su falta de desarrollo, lo cual representó una limitante del estudio.

Otra limitación del estudio fue el no considerar un mayor número de variables sociodemográficas (sexo, capital cultural de las familias, situación laboral) que ayudarán a explicar de mejor manera los resultados encontrados.

\section{REFERENCIAS BIBLIOGRÁFICAS}

Acevedo, M. (2016). Estrategias de aprendizaje, con relación al rendimiento académico y tiempo en alcanzar el grado universitario en enfermería. Tesis de doctorado. España: Universidad de Málaga. Recuperado de https://riuma.uma.es/xmlui/ handle/10630/14346.

Barbero, M., Vila, E. y Suárez, J. (2003). Psicometría. Madrid: Universidad.

Barrios, M. y Frías, M. (2016). Factores que influyen en el desarrollo y rendimiento escolar de los jóvenes de bachillerato. Revista Colombiana de Psicología, 25 (1), 63-82. Recuperado de https://www.redalyc.org/pdf/804/80444652005.pdf.

Becerra, C. (2016). Variables cognoscitivo-motivacionales relacionadas con el rendimiento escolar de estudiantes de bachillerato. Clabes. Tercera Conferencia Latinoamericana sobre el Abandono en la Educación Superior. México: UNAM. Recuperado de https://revistas.utp.ac.pa/index.php/clabes/article/view/870.

Calle, G. (2013). La evaluación de las habilidades del pensamiento crítico asociadas a la escritura digital. Revista Virtual Universidad Católica del Norte, 40, 68-83. Recuperado de https://revistavirtual.ucn.edu.co/index.php/RevistaUCN/article/ viewFile/446/938.

Campos, N., Habiaga, V., Méndez, E. y Presa, T. (2016). El lugar de las Prácticas Pedagógicas Alternativas en la tradición escolar para la protección de trayectorias escolares. Tópos, para un debate de lo educativo, 8. Rivera, octubre de 2016, 41 51. http://ojs.cfe.edu.uy/index.php/rev_topos/article/view/129 8 (8), 91.

Chacón, F., Padial, R., Clements, Y., Breton, S., Cepero, M. y Zurita, F. (2017). Relación entre el rendimiento académico y autoconcepto en jugadoras de baloncesto de categoría cadete en competición nacional extraescolar. Sport TK: revista euroamericana de ciencias del deporte, 6(2), 75-80. Recuperado de https://hdl.handle. net/11441/80488. 
Cohen, L., Manion, L. y Morrison, K. (2011). Research Methods in Education (7th ed.). London: Routledge.

Comisión Europea. (2015). Documento de trabajo de los servicios de la Comisión. Informe sobre España 2015, con un examen exhaustivo relativo a la prevención y la corrección de los desequilibrios macroeconómicos [COM (2015) 85 final 26.2.2015]. Bruselas, Comisión Europea. Recuperado de http://ec.europa.eu/europe2020/pdf/csr2015/cr2015_spain_es.pdf.

Diesktra, R. (2018). Habilidades para la vida: aprendizaje emocional y social. El aprendizaje emocional y social. Errores frecuentes en la educación y tipos de escuelas. Redes (Programa de televisión).

Escudero, A. (2018). Consideraciones Epistemológicas para el estudio de la Sociedad del Conocimiento. IV Congreso Internacional de Investigación en Socioformación y Sociedad del Conocimiento. República Dominicana: CISFOR.

Eurostat. (2015). Sustainable Development Indicators. Social inclusion, Bruselas, Eurostat. http://ec.europa.eu/eurostat/web/sdi/indicators/social-inclusion.

Eurostat. (2016). Education and Training. Statistics illustrated, Bruselas, Eurostat. http://ec.europa.eu/eurostat/web/education-andtraining/statistics-illustrated.

Fuentes, P. (2018). Un acercamiento a los factores que favorecen el aprendizaje de los estudiantes. Estudio de caso en el Centro de Estudios Científicos y Tecnológicos no. 4 "Lázaro Cárdenas", Ciudad de México. Revista Dilemas Contemporáneos: Educación, Política y Valores, VI(1), 1-22. Recuperado de http://files.dilemascontemporaneoseducacionpoliticayvalores.com/200003954-7706477fa8/18.09.12\%20 Un\%20acercamiento\%20a\%20los\%20factores\%20que\%20favorecen\%20el\%20 aprendizaje.pdf.

García, F., y Musitu, G. (2009). AF5: Autoconcepto Forma 5 [AF5: Self-concept form 5] (3nd ed.). Madrid: TEA.

García-Valcárcel, A. y Tejedor, F. (2018). Percepción de los estudiantes sobre el valor de las TIC en sus estrategias de aprendizaje y su relación con el rendimiento. Educación XX1, 20(2), 137-159. https://doi.org/10.5944/educXX1.13447.

Ibarra, E. y Jacobo, H. (2016). La evolución del autoconcepto académico en adolescentes. Revista Mexicana de Investigación Educativa, 21(68), 4570. Recuperado de http://www.scielo.org.mx/pdf/rmie/v21n68/1405-6666rmie-21-68-00045.pdf.

INEE. (2015). Panorama educativo de México. Indicadores del sistema educativo nacional 2014. Educación básica y media superior, México, INEE. Recuperado de http://spdpuebla.net/documentos/P1B113.pdf.

Inglés, C., Martínez- Monteagudo, M., García- Fernández, J., Valle, A. y Castejón, J. (2015). Goal orientation profiles and self-concept of Secondary School Students. Revista de Psicodidáctica, 20(1), 1-14. Recuperado de https://www.researchgate. net/publication/280986065. 
Jaquinet, M., Rivero, M. y Garnache, A. (2016). La motivación en el rendimiento académico de los estudiantes de medicina. Rev Méd Electrón [Internet], 38(6), 910-915. Recuperado de: http://www.revmedicaelectronica.sld.cu/index.php/ rme/article/view/2042/3222.

Juárez, E. (2018). Abandono escolar en bachillerato: Una mirada a las raíces del fenómeno. Revista Red, Caso por Caso, 11, septiembre-diciembre, 1-12. Recuperado de https://www.inee.edu.mx/index.php/655-blog-revista-red-11-2018/blog-revista-red-articulos/3603-abandono-escolar-en-bachillerato-una-mirada-a-las-raicesdel-fenomeno.

Laguna, N. (2017). La autoestima como factor influyente en el rendimiento académico. Tesis de maestría. Colombia: Universidad de Tolima. Recuperado de: http:// repository.ut.edu.co/bitstream/001/2120/1/APROBADO\%20NIRZA\%20MARISOL\%20LAGUNA\%20PROA\%C3\%91OS.pdf.

Marchesi, A. Blanco, R. y Hernández, L. (Coords.). (2015). Avances y desafíos de la educación inclusiva en Iberoamérica. Organización de Estados Iberoamericanos para la Educación, la Ciencia y la Cultura (OEI). España: MAFRE.

Maza, J. (2018). Expectativas profesionales. Un análisis desde las trayectorias escolares de estudiantes universitarios. Revista Dilemas Contemporáneos: Educación, Política y Valores, 2, 1-29. Recuperado de http://files.dilemascontemporaneoseducacionpoliticayvalores.com/200003702-cee10cfdb5/18.1.4\%20Expectativas $\% 20$ profesionales.\%20Un\%20an\%C3\%A1lisis\%20desde.pdf.

OCDE. (2017). Panorama de la educación 2017. Recuperado de http://www.oecd. org/education/skills-beyond-school/EAG2017CN-Mexico-Spanish.pdf.

OCDE. (2019). Higher Education in Mexico: Labour Market Relevance and Outcomes, Higher Education. Paris: OECD Publishing. https://doi. org/10.1787/9789264309432-en.

Parlan, P., Ibnu, S., Rahayu, S. y Suharti, S. (2018). Effects of the Metacognitive Learning Strategy on the Quality of Prospective Chemistry Teacher's Scientific Explanations. International Journal of Instruction, 11(4), 673-688. Recuperado de https:// doi.org/10.12973/iji.2018.11442a.

Pineda, M. y Cerrón, A. (2015). Pensamiento crítico y rendimiento académico. Horizonte de la Ciencia, 5(8), 105-110. https://dialnet.unirioja.es/servlet/ articulo?codigo=5420484.

Román, J. y Gallego, S. (2008). ACRA. Escalas de Estrategias de Aprendizaje (4a . Ed.). España: TEA.

Sánchez-Sandoval, Y. y Verdugo, L. (2016). Desarrollo y validación de la Escala de Expectativas de futuro en la adolescencia. Anales de psicología, 32(2), 545-554. Recuperado de: http://scielo.isciii.es/pdf/ap/v32n2/psicologia_social5.pdf.

Schmidt, V., Messoulam, N., y Molina, F. (2008). Autoconcepto académico en adolescentes de escuelas medias: Presentación de un instrumento para su 
evaluación. Revista Iberoamericana de Diagnóstico y Evaluación - e Avaliação Psicológica, 25(1), 81-106. Recuperado de https://www.redalyc.org/ pdf/4596/459645445005.pdf.

Secretaría de Educación Pública (2012). Reporte de la Encuesta Nacional de Deserción en la Educación Media Superior (ENDEMS). Recuperado de http://www. sems.gob.mx/work/models/sems/Resource/10787/1/images/Anexo_6Reporte_de_ la_ENDEMS.pdf.

Secretaría de Educación Pública (2019). Construye T. México: SEP. Recuperado de http://www.construye-t.org.mx/.

Secretaría de Educación Pública. (2017). Diagnóstico. S243. Programa Nacional de Becas. Agosto 2017. Recuperado de https:/www.gob.mx/cms/uploads/ attachment/file/261718/S243_Programa_Nacional_de_Becas_-_Diagno_stiCO_2017.pdf.

UNESCO. (2015). El futuro del aprendizaje ¿Qué tipo de aprendizaje se necesita en el siglo XXI? (Investigación y prospectiva en educación. Documentos de trabajo). Recuperado de https://unesdoc.unesco.org/ark:/48223/ pf0000242996_spa.

UNICEF (2018). Niños y niñas fuera de la escuela. Fondo de Naciones Unidas para la Infancia: Representación México. Recuperado de https://www.unicef.org/mexico/ spanish/UNICEF_NFE_MX.pdf.

\section{Anexo 1}
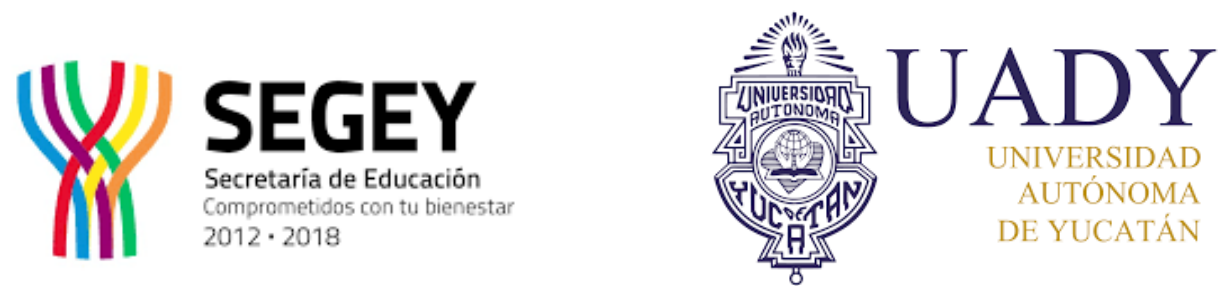

\section{Factores que se constituyen en Barreras para el aprendizaje}

Apreciado Estudiante: Antes que nada queremos agradecerte tu disposición para dar respuesta a este cuestionario el cual nos dará información que nos ayude a identificar los factores que afectan su aprendizaje, dicha información será tratada de manera confidencial, por lo que te pedimos des respuesta a las preguntas de la manera más honesta posible. Es importante aclarar que no existen respuestas buenas o malas, lo único que se busca es conocer la situación real que ustedes viven en la preparatoria. 


\section{Datos generales}

Nombre:

Edad:

Promedio de secundaria:

Escuela preparatoria: Grupo:

Tipo de secundaria donde estudiaste:

¿Reprobaste algún grado de secundaria?

$$
\text { Pública () Privada () Sí () No () }
$$

Número de materias aprobadas en extraordinario durante la secundaria:

¿Estudiaste algún grado de preparatoria en otra escuela?

$$
\text { Sí ( ) No ( ) }
$$

\begin{tabular}{|c|c|c|c|c|c|c|c|c|c|c|c|}
\hline Factor analizado & $\mathbf{0}$ & 1 & 2 & 3 & 4 & 5 & 6 & 7 & 8 & 9 & 10 \\
\hline $\begin{array}{l}\text { 1. Aunque me esfuerce siempre me va mal } \\
\text { en la escuela }\end{array}$ & & & & & & & & & & & \\
\hline $\begin{array}{l}\text { 2. Cometo muchos errores cuando hago mis } \\
\text { tareas }\end{array}$ & & & & & & & & & & & \\
\hline $\begin{array}{l}\text { 3. La mayoría de las actividades que propo- } \\
\text { nen los profesores me estresan }\end{array}$ & & & & & & & & & & & \\
\hline $\begin{array}{l}\text { 4. No puedo hacer bien mis tareas aunque } \\
\text { preste atención a las explicaciones de mis } \\
\text { profesores }\end{array}$ & & & & & & & & & & & \\
\hline $\begin{array}{l}\text { 5. Participo poco en clase porque no tengo } \\
\text { nada importante que aportar }\end{array}$ & & & & & & & & & & & \\
\hline 6. Se me olvida fácilmente lo que aprendo & & & & & & & & & & & \\
\hline $\begin{array}{l}\text { 7. Cuando me concentro puedo estudiar } \\
\text { cualquier cosa }\end{array}$ & & & & & & & & & & & \\
\hline $\begin{array}{l}\text { 8. Soy capaz de participar aportando co- } \\
\text { mentarios o respondiendo a las preguntas } \\
\text { que hacen los profesores }\end{array}$ & & & & & & & & & & & \\
\hline $\begin{array}{l}\text { 9. Soy capaz de entender los diferentes temas } \\
\text { que abordan los profesores durante las clases }\end{array}$ & & & & & & & & & & & \\
\hline $\begin{array}{l}\text { 10. Me considero capaz de competir acadé- } \\
\text { micamente con cualquiera de mis compañe- } \\
\text { ros }\end{array}$ & & & & & & & & & & & \\
\hline $\begin{array}{l}\text { 11. Soy capaz de relacionar mis ideas con } \\
\text { conocimientos adquiridos anteriormente }\end{array}$ & & & & & & & & & & & \\
\hline $\begin{array}{l}\text { 12. Soy capaz de realizar cualquier trabajo } \\
\text { académico que encarguen los profesores }\end{array}$ & & & & & & & & & & & \\
\hline
\end{tabular}

Instrucciones: Marca con una $X$ tu grado de acuerdo con las siguientes afirmaciones, considera que $\mathbf{0}$ significa que estás totalmente en desacuerdo y $\mathbf{1 0}$ que estás totalmente de acuerdo. 


\begin{tabular}{|c|c|c|c|c|c|c|c|c|c|c|c|}
\hline Factor analizado & $\mathbf{0}$ & 1 & 2 & 3 & 4 & 5 & 6 & 7 & 8 & 9 & 10 \\
\hline $\begin{array}{l}\text { 13. Soy un buen alumno, considerando mis } \\
\text { cualidades y conducta en la escuela }\end{array}$ & & & & & & & & & & & \\
\hline $\begin{array}{l}\text { 14. Soy capaz de mantener un buen rendi- } \\
\text { miento a lo largo de todo el semestre }\end{array}$ & & & & & & & & & & & \\
\hline $\begin{array}{l}\text { 15. Busco aprender cosas nuevas para pro- } \\
\text { fundizar después en ellas }\end{array}$ & & & & & & & & & & & \\
\hline $\begin{array}{l}\text { 16. Acostumbro buscar información a favor } \\
\text { y en contra de mis argumentaciones }\end{array}$ & & & & & & & & & & & \\
\hline $\begin{array}{l}\text { 17. Uso cuadros sinópticos, mapas menta- } \\
\text { les, dibujos, etc., para organizar mis ideas }\end{array}$ & & & & & & & & & & & \\
\hline $\begin{array}{l}\text { 18. Busco información necesaria para com- } \\
\text { prender un tema }\end{array}$ & & & & & & & & & & & \\
\hline $\begin{array}{l}\text { 19. Antes de entregar mis tareas, elaboro va- } \\
\text { rias versiones hasta llegar a la que considero } \\
\text { que cumple con lo solicitado }\end{array}$ & & & & & & & & & & & \\
\hline $\begin{array}{l}\text { 20. Subrayo las ideas principales de los tex- } \\
\text { tos que leo }\end{array}$ & & & & & & & & & & & \\
\hline $\begin{array}{l}\text { 21. Busco retroalimentar mis ideas con las } \\
\text { opiniones de otros }\end{array}$ & & & & & & & & & & & \\
\hline $\begin{array}{l}\text { 22. Reviso los argumentos de mis trabajos } \\
\text { antes de redactar las conclusiones }\end{array}$ & & & & & & & & & & & \\
\hline $\begin{array}{l}\text { 23. Analizo continuamente mi proceso de } \\
\text { aprendizaje para identificar lo que debo } \\
\text { mejorar y por qué }\end{array}$ & & & & & & & & & & & \\
\hline $\begin{array}{l}\text { 24. Retomo las ideas de los compañeros } \\
\text { para explicar lo visto en la clase }\end{array}$ & & & & & & & & & & & \\
\hline $\begin{array}{l}\text { 25. Antes de actuar reflexiono sobre las ven- } \\
\text { tajas y desventajas de lo que voy a hacer }\end{array}$ & & & & & & & & & & & \\
\hline $\begin{array}{l}\text { 26. En mis escritos corrijo los errores que } \\
\text { pueden afectar la claridad }\end{array}$ & & & & & & & & & & & \\
\hline $\begin{array}{l}\text { 27. Busco respuestas alternativas a los pro- } \\
\text { blemas que se me plantean }\end{array}$ & & & & & & & & & & & \\
\hline $\begin{array}{l}\text { 28. Me comunico de manera eficaz, re- } \\
\text { flexionando antes de hablar }\end{array}$ & & & & & & & & & & & \\
\hline $\begin{array}{l}\text { 29. Mis decisiones las tomo pensando en la } \\
\text { manera en que éstas influirán en mi futuro }\end{array}$ & & & & & & & & & & & \\
\hline $\begin{array}{l}\text { 30. Como resultado de mis estudios profe- } \\
\text { sionales me compraré un carro }\end{array}$ & & & & & & & & & & & \\
\hline $\begin{array}{l}\text { 31. Considero que terminaré mis estudios de } \\
\text { bachillerato }\end{array}$ & & & & & & & & & & & \\
\hline $\begin{array}{l}\text { 32. Frecuentemente pienso en las cosas que } \\
\text { haré en el futuro }\end{array}$ & & & & & & & & & & & \\
\hline $\begin{array}{l}\text { 33. Como resultado de mis estudios lograré } \\
\text { tener una casa propia }\end{array}$ & & & & & & & & & & & \\
\hline
\end{tabular}

